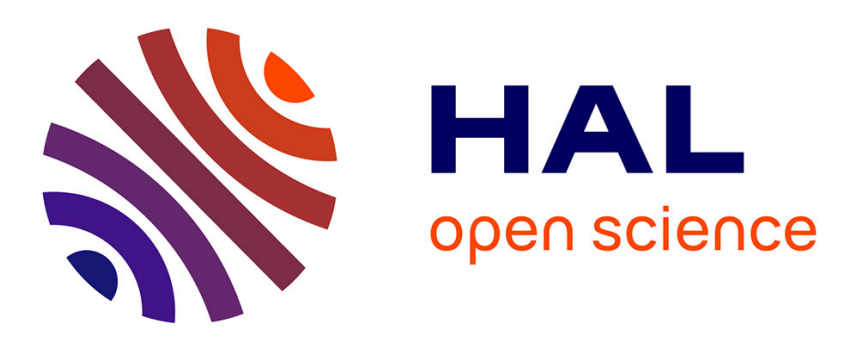

\title{
Magnetic circular dichroism anisotropy of the CuA centre of nitrous oxide reductase from coherent Raman detected electron spin resonance spectroscopy
}

Stephen Bingham, Tim Rasmussen, Jaqui Farrar, Daniel Wolverson, Andrew J Thomson

\section{To cite this version:}

Stephen Bingham, Tim Rasmussen, Jaqui Farrar, Daniel Wolverson, Andrew J Thomson. Magnetic circular dichroism anisotropy of the $\mathrm{CuA}$ centre of nitrous oxide reductase from coherent Raman detected electron spin resonance spectroscopy. Molecular Physics, 2008, 105 (15-16), pp.2169-2176. 10.1080/00268970701732985 . hal-00513154

\section{HAL Id: hal-00513154 \\ https://hal.science/hal-00513154}

Submitted on 1 Sep 2010

HAL is a multi-disciplinary open access archive for the deposit and dissemination of scientific research documents, whether they are published or not. The documents may come from teaching and research institutions in France or abroad, or from public or private research centers.
L'archive ouverte pluridisciplinaire HAL, est destinée au dépôt et à la diffusion de documents scientifiques de niveau recherche, publiés ou non, émanant des établissements d'enseignement et de recherche français ou étrangers, des laboratoires publics ou privés. 


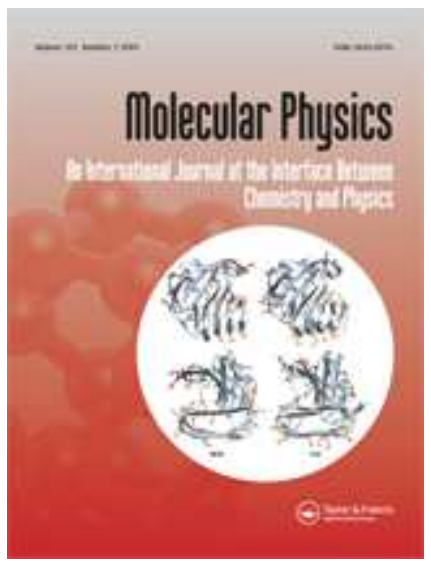

\section{Magnetic circular dichroism anisotropy of the CuA centre of nitrous oxide reductase from coherent Raman detected electron spin resonance spectroscopy}

\begin{tabular}{|c|c|}
\hline Journal: & Molecular Physics \\
\hline Manuscript ID: & TMPH-2007-0185.R1 \\
\hline Manuscript Type: & Full Paper \\
\hline $\begin{array}{r}\text { Date Submitted by the } \\
\text { Author: }\end{array}$ & 31-Aug-2007 \\
\hline Complete List of Authors: & $\begin{array}{l}\text { Bingham, Stephen; University of Bath, Department of Physics } \\
\text { Rasmussen, Tim; University of East Anglia, School of Chemical } \\
\text { Sciences and Pharmacy } \\
\text { Farrar, Jaqui; University of East Anglia, School of Chemical Sciences } \\
\text { and Pharmacy } \\
\text { Wolverson, Daniel; University of Bath, Department of Physics } \\
\text { Thomson, Andrew; University of East Anglia, School of Chemical } \\
\text { Sciences and Pharmacy }\end{array}$ \\
\hline Keywords: & $\begin{array}{l}\text { Magnetic circular dichroism, CuA, Coherent Raman, Electron spin } \\
\text { resonance }\end{array}$ \\
\hline
\end{tabular}

\section{s ScholarONE" \\ Manuscript Central}


Magnetic circular dichroism anisotropy of the $\mathrm{Cu}_{\mathrm{A}}$ centre of nitrous oxide reductase from coherent Raman detected electron spin resonance spectroscopy

\author{
S.J. BINGHAM $* \dagger$, T. RASMUSSEN $\ddagger \S$, J. FARRAR $\ddagger$ D.WOLVERSON $\dagger, \&$
} A.J.THOMSON $\$$

$\dagger$ Department of Physics, University of Bath, Bath, BA2 7AY, UK

$\ddagger$ Centre for Metalloprotein Biology and Spectroscopy, School of Chemical Sciences and Pharmacy, University of East Anglia, Norwich, NR4 7TJ, UK

*Corresponding author. Email: s.j.bingham@bath.ac.uk

§Current address: School of Medical Sciences, University of Aberdeen, Aberdeen, AB25 2ZD, UK

Coherent Raman detected electron spin resonance spectroscopy is a technique that bridges the established fields of magnetic resonance and magneto-optics. By exploiting the orientational selectivity of the microwave resonance condition it becomes possible to measure the relative orientations of the magnetic and optical anisotropies of paramagnetic chromophores, and thereby to test models of their electronic structure. Here we report the application of this method to the $\mathrm{Cu}_{\mathrm{A}}$ centre from Paracoccus pantotrophus nitrous oxide reductase, an unusual mixed valence copper, $\mathrm{Cu}(\mathrm{I}) / \mathrm{Cu}(\mathrm{II})$, dimer centre also found in some heme-copper terminal oxidases. Data from the principal visible bands (at 476, 514 and 750 $\mathrm{nm}$ ) shows that their magnetic circular dichroism is almost entirely aligned with the g-value

1 of 17 
z-axis. This is consistent with previous models of the electronic structure in which the optical transitions are polarised within the copper-thiolate plane of the centre, and the gvalue $\mathrm{z}$-axis is orientated normal to this plane.

Keywords: Magnetic circular dichroism; CuA; Coherent Raman; Electron spin resonance

\section{Introduction}

Nitrous oxide reductase $\left(\mathrm{N}_{2} \mathrm{OR}\right)$ catalyses the final step of bacterial dinitrification, the reduction of nitrous oxide $\left(\mathrm{N}_{2} \mathrm{O}\right)$ to dinitrogen $\left(\mathrm{N}_{2}\right)[1]$ It consists of two copper-containing clusters, one called $\mathrm{Cu}_{\mathrm{Z}}$ being the catalytic centre at which nitrous oxide is reduced to nitrogen, comprising a tetranuclear copper cluster bridged by an inorganic sulphide ion. The other centre, called $\mathrm{Cu}_{\mathrm{A}}$, facilitates electron transfer to the catalytic site in $\mathrm{N}_{2} \mathrm{OR}$ and other complex enzymes such as cytochrome c oxidase (the terminal oxidase of respiration in higher organisms) and a recently identified nitric oxide reductase $[2\lfloor$. The centre contains two copper ions bridged by the thiolate sulphurs of two cysteine amino acid residues, forming an approximately flat rhomboid, figure 1. Each copper ion is additionally coordinated by an imidazole nitrogen from histidine lying close to the rhomboidal plane. Each copper is also relatively weakly coordinated by a longer bond to a forth amino acid residue (one methionine sulphur, and one backbone carbonyl oxygen in $\mathrm{N}_{2} \mathrm{OR}$ ) forming a highly distorted tetrahedral coordination geometry around both coppers. The electron

Deleted: each transfer functionality of the centre is achieved by redox cycling between a reduced diamagnetic di-cuprous state and an oxidised paramagnetic mixed valence state in which the unpaired electron is essentially equally delocalised between the two copper ions. This

2 of 17 
delocalised state was first identified from the fact that the single unpaired electron interacts almost equally with each copper nucleus. The individual nuclear spins of $3 / 2$ give a characteristic combined seven-line hyperfine pattern that is often particularly well resolved in the g-parallel region of the electron spin resonance "powder" spectrum of the frozen aqueous solution.

The unusual electronic structure of $\mathrm{Cu}_{\mathrm{A}}$ has attracted study by many spectroscopic methods [11. Low temperature magnetic circular dichoism (MCD) studies in particular have identified the principal optical transitions in the near-UV to near-IR region $[3,4 \downarrow$, The dominant feature is a pair of oppositely signed temperature dependent MCD bands centred at about $500 \mathrm{~nm}\left(20,000 \mathrm{~cm}^{-1}\right)$. Such a feature, which is known as a "pseudo A-term" in the MCD literature, is characteristic of two transitions with mutually perpendicular linear polarisation and similar energy whose states have been strongly mixed by spin-orbit coupling. The second main feature is a negative MCD band at about $750 \mathrm{~nm}\left(13300 \mathrm{~cm}^{-1}\right)$. In order to probe the nature of the optical transitions, and hence the electronic structure, in more detail we would like to measure the orientational dependence of the MCD. Such information is, in principle, contained within the magnetic field and temperature dependence of the MCD of a frozen aqueous solution, since centres with different orientations with respect to the applied field have different g-values and hence different MCD field/temperature dependences [5-7l. However, in species with relatively small gvalue anisotropies, such as copper centres, the analysis of such MCD "saturation" or "magnetisation" curves leads to low accuracy since it involves deconvolution of the data with a continuous distribution of very similar simulation functions.

Deleted: [5-7]

3 of 17 
A much more accurate method exploits the high spectral resolution of electron spin resonance (ESR). Since g-value anisotropy is often well resolved in an ESR experiment, we can use the microwave resonance condition to select centres with specific orientations with respect to the applied magnetic field. If we can use microwave resonance to modulate or perturb the MCD, we can perform an orientationally selective MCD experiment in which the relative orientations of the MCD and g-value anisotropies can, in principle, be deduced. Essentially the same fundamental approach is widely used in a variety of electron-nuclear spectroscopies to obtain orientationally resolved measurements.

An early implementation of this microwave-selected MCD idea built upon established optically detected magnetic resonance principles $[8-11]$. These experiments use MCD to monitor changes in the spin polarisation (population difference) of the ESR transition that occur when microwave power is absorbed. Two difficulties were encountered with this approach, however. Firstly, a variety of complex magnetic relaxation phenomena [11] (cross-relaxation and spectral diffusion) tend to remove the orientational selectivity of the experiment since these processes can change the spin polarisation of centres that are not resonant with the applied microwave field [12]. It is quite possible for "inhomogenously broadened" ESR spectra to microwave power saturate "homogenously". Additionally, the signal magnitude in this experiment is determined by the balance between the longitudinal relaxation phenomena (spin-lattice relaxation, cross relaxation and spectral diffusion) and the microwave power absorption. Simulation of data to extract MCD anisotropy therefore requires knowledge of the anisotropy (and magnetic field dependence) of these magnetic relaxation processes. Frequently such data are not available. Unravelling the anisotropies of the magnetic relaxation and the MCD is especially difficult in proteins containing multiple

Deleted: [8-11]

Deleted: [11]

Deleted: [12]

Deleted: is

Deleted: 20

Deleted: 18 
paramagnetic centres, and samples with unknown quantities of minor paramagnetic species. In some early work it was simply assumed that the magnetic relaxation was isotropic and independent of magnetic field [8,9l, Despite these difficulties, these "longitudinally

Deleted: $[8,9]$ detected" experiments did show success in the deconvolution of overlapping optical spectra due to multiple chemical species. These experiments relied, in effect, on distinguishing between the different centres by virtue of their different microwave power saturation characteristics. A particularly important example, and one especially relevant here, was the separation of the $\mathrm{Cu}_{\mathrm{A}} \mathrm{MCD}$ spectrum from the intense heme spectrum of cytochrome $\mathrm{c}$ oxidase [9]. This experiment was one of the first to clearly identify the principle features of the $\mathrm{Cu}_{\mathrm{A}}$ described above, although with the benefit of hindsight some minor features may be due to perturbation of the heme MCD via magnetic interaction (cross-relaxation) with the $\mathrm{Cu}_{\mathrm{A}}$ rather than the $\mathrm{Cu}_{\mathrm{A}}$ itself.

The magnetic relaxation related uncertainties encountered with the longitudinally detected experiment led to a fundamental revision of the measurement strategy [12l, It is well known that the signal magnitude in conventional ESR spectroscopy, when integrated over the spectral lineshape, is independent of the magnetic relaxation processes [13] Interaction with other paramagnetic centres, and other interactions with the environment, leads to changes in the ESR lineshape only. This arises from the fact that the precessing magnetisation perpendicular to the applied field is recorded, rather than changes in the magnetisation along the field, or, in other words, "coherence" in the spectroscopic transition is recorded rather than spin polarisation of the stationary eigenstates. The equivalent experiment in the MCD context is the measurement of the sinusoidally oscillating MCD perpendicular to the applied field. The removal of the experiment's 
dependence on magnetic relaxation phenomena causes a vast simplification in the interpretation and simulation. We may, for example, assume that different paramagnetic species in the sample contribute separately and linearly to the observed spectrum. Such a basic property is taken for granted in conventional ESR. Like conventional ESR we may also accurately simulate data from knowledge of the matrix elements between the (effective) spin states, other interactions and degrees of freedom can be subsumed within phenomenological lineshape functions $[14 \mathrm{l}$

Deleted: [14]

The measurement of the microwave induced precession of the MCD offers unprecedented orientational and chemical selectivity. However, this selectivity also brings a considerable metrology challenge. Only a very small proportion of the chromophores present in the sample interact resonantly with the microwaves, but they may all contribute to the optical absorption. The microwave modulation of the MCD in a typical experiment is therefore often only a very small proportion of the total conventional MCD of the sample. Typical signal magnitudes in metallo-enzyme samples are a hundred times smaller than the noise floor of the spectropolarimeters typically used in MCD spectroscopy. The single previous example of an ESR measurement detected by a microwave modulated magneto-optical effect (on a single crystal of CdS) [15] involved modulations about five orders of magnitude larger than a metallo-enzyme experiment. Whereas these unusually large modulations can be detected by spectrally resolving the modulation side-bands using laser interferometry $[15,16]$ it is highly improbable that this approach would be feasible for biological samples. Instead a method that had previously been used for radiofrequency spectroscopy of atomic vapours [17 was extended to the microwave region - optical heterodyne detection. The development of efficient photodetectors with microwave

Deleted: $[15,16]$

Deleted: [17]

Deleted: 20

Deleted: 18 
frequency response bandwidths enabled this method to achieve near quantum limited detection sensitivity using a few $\mathrm{mW}$ of visible laser light [18] Crucial in achieving this Deleted: [18] performance is the fact that optical heterodyne detection, unlike laser interferometry, is essentially blind to fluorescence and other "incoherent" optical backgrounds. The first instrument of this type was constructed by one of us in 1995 at the Department of Physics, ETH, Zürich. This endeavour combined equipment and expertise from both the University of East Anglia, Norwich, UK and ETH. In this special edition of Molecular Physics dedicated to the memory of Arthur Schweiger (ETH), we recall his enthusiastic and generous support of the project, without which it is unlikely that instrument could have been built. Using this prototype instrument it was possible to demonstrate the first experiment on paramagnetic transition ions, namely, $\mathrm{Cr}^{3+}$ in Ruby [19].

The subsequent development and refinement of the microwave modulated MCD technique centred initially on its application to metallo-proteins $[14,20,21]$ (with collaborators at the University of Dortmund) and more recently on its uses in solid-state physics studies [2224l. In some contexts it is more straightforward to think of the experiment as an optically detected ESR experiment rather than a microwave modulated MCD experiment as we have emphasised here. It is also useful to recognise that the creation of microwave frequency modulation sidebands about a central laser excitation frequency can also be formulated in terms of coherent Raman processes [25], For these reasons we follow the convention in the physics literature and refer to the experiment as a coherent Raman detected ESR experiment.

When the measurement of ESR is the principal goal of an experiment, coherent Raman detection can, like other optically detected magnetic resonance (ODMR) techniques[10, 26, 
27], offer dramatically improved detection sensitivity over standard microwave detection. The chemical selectivity provided by optical detection is very powerful in many applications. Coherent Raman detection complements the better known luminescence detected ODMR methods in that it principally targets magnetic resonance in the electronic ground level. The simplification of theoretical interpretation and maintenance of resonant selectivity that result from the detection of the precessing spin polarisation (magnetisation) are also often crucially important in the ESR detection context. Finally we note that a form of coherent Raman detected ESR experiment exists, "Raman heterodyne detected ESR", that is related to optical hole-burning techniques rather than the MCD related technique described here[28]. Its feasibility is determined by relatively unusual optical relaxation phenomena being present, and the quantitative interpretation of the experiment requires a detailed knowledge of these processes.

\section{Theory}

The theory of coherent Raman detected ESR of an isolated Kramers doublet (an effective spin $1 / 2$ system) has been described in detail elsewhere [14L, Only the underlying principles will be outlined here. A useful starting point is to recognise that the optical linewidths of almost all condensed phase materials, including those of the sample studied here, are considerably larger than the microwave frequency employed in a coherent Raman detected ESR experiment. This means that we can decouple the microwave frequency dynamics from the optical frequency dynamics, and the latter need therefore not be explicitly considered. In these circumstances, it is meaningful to talk about changes in optical absorption and dispersion on a timescale comparable to the microwave precession period.

8 of 17 
In addition we can note that magnetic circular dichroism has the same fundamental symmetry as a magnetic dipole - both are time-odd axial vectors. These facts mean that we can visualise the experiment as a MCD vector that precesses (and nutates) in response to an applied microwave field in the same way as the magnetic moment responds. In a simple isotropic (atomic) case $[29]$ the MCD vector precesses about the main magnetic field. The phase of the precession relative to the oscillating microwave field is the same as that of the magnetic moment in the conventional ESR experiment, and the amplitude of the precessing MCD and the magnetic moments are proportional to each other. The two quadrature phase components of the precessing MCD are analogous to the conventional ESR absorption and dispersion signals [13]. This picture also makes it easy to understand that measurement of the precessing MCD is best achieved using a circularly polarised light beam propagating perpendicularly to the applied magnetic field.

Naturally the anisotropic case is more complex. It is conveniently approached by considering the motion of the (effective or fictitious) spin vector $\bar{S}$ and expressing the MCD in terms of this spin: $\overline{M C D} \propto \overline{\bar{C}} \cdot \bar{S}$, where $\overline{\bar{C}}$ is a matrix relating the Cartesian components of the MCD vector, $\overline{M C D}$, to those of the effective spin vector. This is directly analogous to the magnetic dipole case: $\bar{M} \propto \bar{g} \cdot \bar{S}$, where $\overline{\bar{g}}$ is the g-matrix (or "tensor") commonly used in ESR spectroscopy [30l, In the anisotropic case the effective spin vector $\bar{S}$ of an individual molecule will still precess in response to the applied microwave field, but it will do so about an axis other than the main applied field. More subtly, the phase of the precession, observed in the laboratory frame, will also be affected by the magnetic anisotropy. An analytical solution of the motion of the effective spin vector

Deleted: [27]

9 of 17 
is possible however [14], and the resulting oscillating MCD can therefore also be obtained

in terms of the elements of the matrix $\overline{\bar{C}}$. The components of $\overline{\bar{C}}$ can be related to the electric dipole matrix elements of the monitored optical transition:

$C_{i l}(A \rightarrow J)=\left\langle A, l\left|C_{i}(A \rightarrow J)\right| A, l\right\rangle ; \quad C_{i}(A \rightarrow J)=\frac{1}{2 i} P_{A}\left(m_{j} P_{J} m_{k}-m_{k} P_{J} m_{j}\right) P_{A}$

where $l=x, y, z$ are principal axes of the g-matrix; $i, j, k=x, y, z$ and symmetric permutations of these indices; $|A, l\rangle$ is a state of the ground level manifold A with fictitious spin quantised along the lth g-value axis; $C_{i}(A \rightarrow J)$ is an operator describing the paramagnetic ("C-term" [31]) MCD of the absorption band between manifolds A and J, observed along the ith g-value axis; $m_{j}$ is the electric dipole operator along the jth g-value axis; and $P_{J}=\sum_{l}|J, l\rangle\langle J, l|$ is a projection operator into manifold $\mathrm{J}$ (and similarly for $P_{A}$ ). In the absence of molecular spatial symmetry, all nine elements of the matrix, $\overline{\bar{C}}$, will be non-zero, however in a sample containing randomly orientated molecules only the three diagonal elements are observable: $C_{x x}, C_{y y}$ and $C_{z z}$. Although contributions arise from the off-diagonal elements for individual molecules, another molecular orientation will always exist that gives rise to an equal and opposite cancelling contribution. From the form of the operator $C_{i}(A \rightarrow J)$ we can see that an MCD along the ith g-value axis requires non-zero electric dipoles along both axes orthogonal to it ( $\mathrm{j}$ and $\mathrm{k}$ ). This arises from the need to couple to circularly polarised radiation, which has fields in both directions orthogonal to the propagation axis.

10 of 17 


\section{Experimental section}

The sample of nitrous oxide reductase was purified from Paracoccus pantotrophus under anaerobic conditions, then oxidised using ferricyanide as described in detail elsewhere [3]. The optical absorbance, electron spin resonance and magnetic circular dichroism spectra of this form of the enzyme have also been reported elsewhere [3] and support the view that the primary paramagnetic species is the $\mathrm{Cu}_{\mathrm{A}}$, the catalytic $\mathrm{Cu}_{Z}$ centre being primarily in a diamagnetic oxidised form. The sample was dissolved in $10 \mathrm{mM} \mathrm{Na}$ HEPES zwitterionic buffer at $\mathrm{pH} 7.0$ and mixed with an equal volume of glycerol so that an optical transparent glass was formed on freezing. The protein concentration was estimated by the BCA method to be $2.13 \mathrm{mM}$ of protein dimer prior to dilution with glycerol. Chemical analysis identified 11.2 $\mathrm{Cu}$ and 1.5 labile sulphides per dimer.

Coherent Raman spectra were measured with an instrument described in detail elsewhere $[18,19,21,22]$. A visible continuous-wave laser beam passes through the sample along an axis perpendicular to the applied magnetic field provided by a split-coil superconducting magnet system. Strain-free mounted low-temperature windows allow the polarisation of the light at the sample to be controlled while allowing the temperature to be reduced to approximately $1.4 \mathrm{~K}$ by emersion in a superfluid liquid helium bath. A microwave frequency (approx 13.8 GHz) magnetic field is applied parallel to the propagation axis of the laser beam, and its magnitude is enhanced by surrounding the sample by a high $\mathrm{Q}$ resonant cavity similar to that employed in conventional ESR spectroscopy [32] For reasons outlined in section 2, a circularly polarised laser beam will be amplitude modulated at the microwave frequency by the precessing spin in this geometry. This modulation is

11 of 17

Deleted: Helium

Deleted: [29]

Deleted: 20

Deleted: 18 
detected with a high-speed InGaAs photodiode and the resultant microwave frequency signal is detected using a low noise microwave receiver. This detection system achieves near shot/quantum noise limited sensitivity under the conditions of these measurements.

Since the microwave signal generated by the photodiode is phase-coherent with the sample-excitation oscillator we can measure the phase of the signal using the latter as a reference for a phase-sensitive microwave detector. The microwave detection circuit is closely analogous to the "homodyne bridge" circuits commonly employed in ESR spectroscopy [32l and it is therefore possible to measure the analogues of both the absorption and dispersion phase ESR signals. In coherent Raman detected ESR it is usually optimal to measure dispersion phase spectra at relatively high microwave irradiation levels and relatively low temperatures compared to conventional ESR. In solid-state samples the dispersion phase signal does not power saturate as easily as the absorption phase signal, and can be measured with equal sensitivity in the coherent Raman detected instrument.

Changing the polarisation of the light from left to right circularly polarised changes the sign of the microwave frequency modulation. It is therefore convenient and efficient to use a photo-elastic polarisation modulator [33 to provide a secondary $50 \mathrm{kHz}$ signal modulation that can be processed using conventional DSP based lock-in amplifiers. This latter modulation performs a similar noise/drift suppression role as the magnetic field modulation commonly employed in conventional ESR spectroscopy [32l, Because we do not use field modulation we record and present the data as the absolute signal rather than its first derivative with respect to field as is common in ESR.

12 of 17 


\section{Results and analysis}

The low temperature MCD of this form of the enzyme shows dominant $\mathrm{Cu}_{\mathrm{A}}$ features at

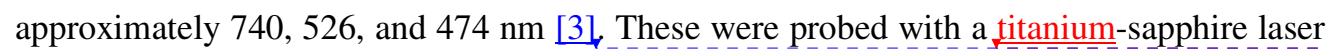
$(750 \mathrm{~nm})$ and two lines from an argon ion laser $(514 \mathrm{~nm}, 476 \mathrm{~nm})$. Dispersion phase spectra Deleted: [3] Deleted: Titanium Deleted: Argon measured at these wavelengths are shown in Figure 2. Since the MCD is negative in the 750 and $476 \mathrm{~nm}$ regions the coherent Raman detected spectra have opposite sign to the 514 $\mathrm{nm}$ spectrum. It is immediately obvious that the 476 and $514 \mathrm{~nm}$ spectra have very similar shapes compared to the $750 \mathrm{~nm}$ spectrum. There was no evidence of microwave power dependent changes in the lineshape.

Quantitative analysis of the MCD anisotropy requires comparison with computer simulated lineshape functions. In order to minimise the number of free parameters we first simulate the conventional X-band $(9.64 \mathrm{GHz})$ ESR spectrum, figure 3. We choose to simulate the microwave frequency susceptibility rather than the experimentally recorded first derivative with respect to magnetic field. It is the overall lineshape, rather than subtle hyperfine related effects, that is important here. Our program includes copper hyperfine but assumes that the interaction is co-axial with the g-matrix. It also includes isotropic g-strain $[341$ through a Gaussian distribution of g-values. The spin Hamiltonian and line broadening parameters required to fit the data are very close to those needed for other $\mathrm{Cu}_{\mathrm{A}}$ centres $[35]$ figure 3 .

Coherent Raman spectra are calculated using the same program used to calculate the conventional ESR spectrum, figure 4. Only the microwave frequency has been adjusted to that of the coherent Raman experiment $(13.68 \mathrm{GHz})$, other parameters remain unchanged

13 of 17 
from the ESR simulation. The explicit expressions for the MCD modulation that need to be integrated by the program are given in reference [14]. Unlike many conventional ESR simulation programs the dispersion phase signal is calculated as well as the absorption phase signal, and because of this it must take into account the response of the system to both positive and negative microwave frequencies [14]. In the general case a spectrum will be a linear combination of three contributions from $C_{x x}, C_{y y}$ and $C_{z z}$. However in the present sample the g-matrix is nearly axial - the $g_{x}$ and $g_{y}$ regions of the spectrum cannot be individually resolved. This means that the $C_{x x}$ and $C_{y y}$ components cannot be separated. Instead we consider their average: $C_{\perp}=\left(C_{x x}+C_{y y}\right) / 2$. Figure 4 shows the simulated spectra arising from the $C_{z z}$ and $C_{\perp}$ contributions. The conventional ESR spectrum (figure 4c) is also provided for comparison.

The data, figure 2, are fitted by changing the relative proportion of the $C_{z z}$ and $C_{\perp}$ spectra. These contributions need not have the same sign, but do so in this case. The data clearly shows that for the 514 and $476 \mathrm{~nm}$ bands the MCD is almost entirely due to the $C_{z z}$ contribution. Residual errors between the data and the fits are remarkably small, but do result in a small amount of ambiguity. We also looked for correlations between the ESR fitting parameters and the MCD anisotropy parameters. Overall an accuracy in the ratio of $C_{z z}$ and $C_{\perp}$ of around +/- 0.05 is conservatively estimated from this data. This is an order of magnitude better than could realistically be obtained by the analysis of MCD saturation/magnetisation curves in samples such as these [5-71. This accuracy is well illustrated by the fact that the $750 \mathrm{~nm}$ spectrum has a significantly larger $C_{\perp}$ contribution,

14 of 17
Deleted: [14]

Deleted: [14]
Deleted: [5-7]

Deleted: 20

Deleted: 18 
compared to the other two bands. Although clear in this data, a MCD saturation/magnetisation curve analysis would be unable to distinguish between the anisotropy at these three wavelengths.

\section{Discussion}

The results presented here are, to a first approximation, consistent with a relatively simple model for the $\mathrm{Cu}_{\mathrm{A}}$ centre described previously [4]. This calculation approximated the centre to an ideally symmetrical and planar $\mathrm{D}_{2 \mathrm{~h}}$ structure. The MCD is accounted for by four dominant transitions. The $476 \mathrm{~nm}$ region is a $a_{g} \rightarrow b_{3 u}$ one electron excitation (the $b_{3 u}$ state being the highest occupied orbital of the ground level) polarised along the $\mathrm{Cu}-\mathrm{Cu}$ axis. The $514 \mathrm{~nm}$ region is due to a $b_{1 g} \rightarrow b_{3 u}$ transition polarised along the orthogonal S-S axis. Since these transitions are close in energy, strong mixing by spin-orbit coupling results in a "pseudo-A term" pattern of equally intense and oppositely signed MCD bands. Since neither of the un-mixed transitions contain any transition dipole polarisation perpendicular to the plane of the rhomboid an MCD can only be generated along an axis normal to the rhomboid. The $750 \mathrm{~nm}$ region is accounted for by two transitions in the same model which have the same symmetries as the high energy bands just described. However in this case $\underline{a}$ symmetrical "pseudo-A term" pattern is not present. This is accounted for by the bulk of the MCD intensity arising from mixing of a low lying $B_{2 u}$ level into the $B_{3 u}$ ground level by spin-orbit coupling, rather than the mixing of the excited $A_{g}$ and $B_{1 g}$ levels [4]. Despite its different origin, the MCD of these transitions can also only occur along an axis normal to the rhomboid plane since the transitions $B_{2 u} \rightarrow A_{g}$ and $B_{2 u} \rightarrow B_{1 g}$ are polarised along 
the $\mathrm{S}-\mathrm{S}$ and $\mathrm{Cu}-\mathrm{Cu}$ axes respectively. Finally, the mixing of the $B_{2 u}$ level into the $B_{3 u}$ ground level by spin-orbit coupling is also the main mechanism by which g-value । anisotropy is created. The mixing of copper $d_{x^{2}-y^{2}}$ and $d_{x y}$ orbital character, and also I sulphur $p_{x}$ and $p_{y}$ orbital character, by this mechanism results in an orbital enhancement of the ground level g-value normal to the rhomboid plane. Thus the g-value $\mathrm{z}$-axis lies perpendicular to the rhomboid plane in this model, and, because all the optical transitions I involved are polarised in the plane, the MCD will be principally normal to the plane and | parallel to the g-value z-axis, as observed. The observation of small amounts of MCD in the g-value $x-y$ plane requires some mixing of states giving rise to a transition dipole along the g-value z-axis. Further mixing by spin-orbit coupling, or the breakdown of the idealised $D_{2 h}$ symmetry would allow this. Comparison with an accurate electronic structure calculation is therefore the next phase required in this work.

\section{Acknowledgments}

The support of the UK EPSRC and BBSRC research councils is gratefully acknowledged.

\section{References}

16 of 17
Deleted: [1] . W. G. Zumft, P. M. H Kroneck, Advances in Microbial Physiology, Vol. 52, pp. 107, Academic press, London (2007)प

[2] . Suharti, M. J. F. Strampraad, I. Schroder, S. de Vries, Biochemistry, 40, 2632 (2001)I

[3] . T. Rasmussen, B. C. Berks, J. N. Butt, A. J. Thomson, Biochem. J., 364 807 (2002)【

[4] J. A. Farrar, F. Neese, P.

Lappalainen, P. M. H. Kroneck, M. Saraste, W. G. Zumft, A. J. Thomson, $J$.

Am. Chem. Soc., 118, 11501 (1996)d

[5] . A. J. Thomson, M. K. Johnson, Biochem. J., 191, 411 (1980)प

[6] . F. Neese, E. I. Solomon, Inorg. Chem., 38, 1847 (1999)II

[7] . A. J. Thomson, M. R. Cheesman, S. J. George, Methods Enzymol., 226, 199 (1993)II

[8] . C. P. Barrett, J. Peterson, C. Greenwood, A. J. Thomson, J. Am. Chem. Soc., 108, 3170 (1986)प [9] . A. J. Thomson, C. Greenwood, J. Peterson, C. P. Barrett, J. Inorg. Biochem., 28, 195 (1986)【

[10] . S. Geschwind, In Electron Paramagnetic Resonance, S. Geschwind (Ed.), Plenum, New York (1972)【I

[11] . K. J. Standley, R. A. Vaughan, Electron Spin Relaxation Phenomena in Solids, Adam Hilger, London (1969)【I [12] . S. J. Bingham, Magnetic circular dichroism and electron paramagnetic resonance of transition ions, $\mathrm{Ph} . \mathrm{D}$. thesis, University of East Anglia (1993)II [13] . C. P. Slichter, Principles of Magnetic Resonance, 1st ed., Harper, New York (1963)【

[14] . S. J. Bingham, J. Gutschank, B. Borger, D. Suter, A. J. Thomson, J. Chem. Phys., 113, 4331 (2000)I

[15] . R. Romestain, S. Geschwind, G. E. Devlin, P. A. Wolff, Phys. Rev. Letters, 33, 10 (1974)【

[16] . A. S. Borovik-Romanov, N. M. Kreines, Phys. Rep., 81, 351 (1982)प [17] . R. Bernheim, Optical pumping, Benjamin, New York (1965)【

[18] . S. J. Bingham, B. Börger, D. Suter, A. J. Thomson, Rev. Sci. Instrum., 69 , 3403 (1998)प

[19] S. J. Bingham, D. Suter, A. Schweiger, A. J. Thomson, Chem. Phys. Letters, 266, 543 (1997)II

[20] . B. Börger, S. J. Bingham, J. Gutschank, M.-O. Schweika, D. Suter, A. J. Thomson, J. Chem. Phys., 111, 8565 (1999)I

[21] S. J. Bingham, B. Börger, J. Gutschank, D. Suter, A. J. Thomson, $J B I C, \mathbf{5}, 30(2000)$ पI

[22] . S. J. Bingham, J. J. Davies, [.. [1] 


\section{Figure captions}

\section{Figure 1}

Basic chemical structure of the mixed valence $\mathrm{Cu}_{\mathrm{A}}$ centre. The relatively strong nitrogen and sulphur ligation of the copper ions from cysteine and histidine amino acid residues lie symmetrically in a plane. The weaker coordination from a methionine sulphur and a carbonyl oxygen occur from the opposite sides of this plane.

\section{Figure 2}

Coherent Raman detected electron spin resonance spectra of the principal bands of the $\mathrm{Cu}_{\mathrm{A}}$ centre measured in dispersion phase (lines). Simulations (crosses) are labelled with the ratio of the magnetic circular dichroism (MCD) parameter parallel and perpendicular to the g-value z-axis. The 476 and $750 \mathrm{~nm}$ spectra are of opposite sign to the $514 \mathrm{~nm}$ spectrum, and that conventionally used in conventional ESR, because the MCD is negative for these bands.

Figure 3

Conventional X-band $(9.64 \mathrm{GHz})$ electron spin resonance spectra of the $\mathrm{Cu}_{\mathrm{A}}$ centre. Absolute absorption phase susceptibility (upper) and the experimentally recorded first-derivative with respect to magnetic field (lower) are shown. The characteristic seven-line copper hyperfine is visible in the latter. A simulation of the absolute spectrum (dotted line) used g-values of 2.18, 2.025 and 2.02 and coaxial hyperfine splittings of 40 and 22 Gauss.

\section{Figure 4}

Simulated coherent Raman detected electron spin resonance spectra at the frequency $(13.68 \mathrm{GHz})$ of the coherent Raman experiment. Absorption (dotted lines) and dispersion (solid lines) phase spectra are shown. Experimental spectra may be fitted using a linear combination of the two simulations shown. The $\mathrm{C}_{\mathrm{zz}}$ and $\mathrm{C}_{\text {perp }}$ contributions result from an MCD parallel and perpendicular to the g-value z-axis. A simulation of the corresponding conventional microwave detected ESR spectra is also shown for comparison.

17 of 17 
Page 16: [1] Deleted stephen bingham

8/20/2007 3:30:00 PM

[1] W. G. Zumft, P. M. H. Kroneck, Advances in Microbial Physiology, Vol. 52, pp. 107, Academic press, London (2007)

[2] Suharti, M. J. F. Strampraad, I. Schroder, S. de Vries, Biochemistry, 40, 2632 (2001)

[3] T. Rasmussen, B. C. Berks, J. N. Butt, A. J. Thomson, Biochem. J., 364, 807 (2002)

[4] J. A. Farrar, F. Neese, P. Lappalainen, P. M. H. Kroneck, M. Saraste, W. G. Zumft, A. J. Thomson, J. Am. Chem. Soc., 118, 11501 (1996)

[5] A. J. Thomson, M. K. Johnson, Biochem. J., 191, 411 (1980)

[6] F. Neese, E. I. Solomon, Inorg. Chem., 38, 1847 (1999)

[7] A. J. Thomson, M. R. Cheesman, S. J. George, Methods Enzymol., 226, 199 (1993)

[8] C. P. Barrett, J. Peterson, C. Greenwood, A. J. Thomson, J. Am. Chem. Soc., 108, 3170 (1986)

[9] A. J. Thomson, C. Greenwood, J. Peterson, C. P. Barrett, J. Inorg. Biochem., 28, 195 (1986)

[10] S. Geschwind, In Electron Paramagnetic Resonance, S. Geschwind (Ed.), Plenum, New York (1972)

[11] K. J. Standley, R. A. Vaughan, Electron Spin Relaxation Phenomena in Solids, Adam Hilger, London (1969)

[12] S. J. Bingham, Magnetic circular dichroism and electron paramagnetic resonance of transition ions, Ph.D. thesis, University of East Anglia (1993)

[13] C. P. Slichter, Principles of Magnetic Resonance, 1st ed., Harper, New York (1963)

[14] S. J. Bingham, J. Gutschank, B. Borger, D. Suter, A. J. Thomson, J. Chem. Phys., 113, 4331 (2000)

[15] R. Romestain, S. Geschwind, G. E. Devlin, P. A. Wolff, Phys. Rev. Letters, 33, 10 (1974)

[16] A. S. Borovik-Romanov, N. M. Kreines, Phys. Rep., 81, 351 (1982)

[17] R. Bernheim, Optical pumping, Benjamin, New York (1965)

[18] S. J. Bingham, B. Börger, D. Suter, A. J. Thomson, Rev. Sci. Instrum., 69, 3403 (1998)

[19] S. J. Bingham, D. Suter, A. Schweiger, A. J. Thomson, Chem. Phys. Letters, 266, 543 (1997)

[20] B. Börger, S. J. Bingham, J. Gutschank, M.-O. Schweika, D. Suter, A. J. Thomson, J. Chem. Phys., 111, 8565 (1999)

[21] S. J. Bingham, B. Börger, J. Gutschank, D. Suter, A. J. Thomson, JBIC, 5, 30 (2000)

[22] S. J. Bingham, J. J. Davies, D. Wolverson, Phys. Rev. B, 65, art. no. 155301 (2002)

[23] L. C. Smith, D. Wolverson, S. J. Bingham, J. J. Davies, Physica Status Solidi BBasic Solid State Physics, 243, 892 (2006)

[24] L. C. Smith, S. J. Bingham, J. J. Davies, D. Wolverson, Appl. Phys. Lett., 87, Art. No. 202101 (2005)

[25] Y. R. Shen, The Principles of Nonlinear Optics, 1st ed., Wiley, New York (1984)

[26] R. H. Clarke (Ed.), Triplet state ODMR spectroscopy:Techniques and applications to biophysical systems, Wiley, New York (1982)

[27] A. J. Hoff, In Metallobiochemistry, Part D, Methods in Enzymology, Vol. 227, pp. 290, Academic press, San Diego (1993)

[28] K. Holliday, X. F. He, P. T. H. Fisk, N. B. Manson, Optics Letters, 15, 983 (1990) 
[29] H. G. Dehmelt, Phys. Rev., 105, 1924 (1957)

[30] A. Abragam, B. Bleaney, Electron Paramagnetic Resonance of Transition Ions, Oxford University Press, Oxford (1970)

[31] P. J. Stephens, Adv. Chem. Phys., 35, 197 (1976)

[32] T. H. Wilmshurst, Electron Spin Resonance Spectrometers, Adam Hilger, London (1967)

[33] K. W. Hipps, G. A. Crosby, J. Phys. Chem, 83, 555 (1979)

[34] W. R. Hagen, J. Mag. Res., 44, 447 (1981)

[35] F. Neese, W. G. Zumft, W. E. Antholine, P. M. H. Kroneck, J. Am. Chem. Soc., 118, 8692 (1996)

[1] W. G. Zumft, P. M. H. Kroneck, In Advances in Microbial Physiology, Vol. 52, pp. 107, Academic press, London (2007)

[2] Suharti, M. J. F. Strampraad, I. Schroder, S. de Vries, Biochemistry, 40, 2632 (2001)

[3] T. Rasmussen, B. C. Berks, J. N. Butt, A. J. Thomson, Biochem. J., 364, 807 (2002)

[4] J. A. Farrar, F. Neese, P. Lappalainen, P. M. H. Kroneck, M. Saraste, W. G. Zumft, A. J. Thomson, J. Am. Chem. Soc., 118, 11501 (1996)

[5] A. J. Thomson, M. K. Johnson, Biochem. J., 191, 411 (1980)

[6] F. Neese, E. I. Solomon, Inorg. Chem., 38, 1847 (1999)

[7] A. J. Thomson, M. R. Cheesman, S. J. George, Methods Enzymol., 226, 199 (1993)

[8] C. P. Barrett, J. Peterson, C. Greenwood, A. J. Thomson, J. Am. Chem. Soc., 108, 3170 (1986)

[9] A. J. Thomson, C. Greenwood, J. Peterson, C. P. Barrett, J. Inorg. Biochem., 28, 195 (1986)

[10] S. Geschwind, In Electron Paramagnetic Resonance, S. Geschwind (Ed.), Plenum, New York (1972)

[11] K. J. Standley, R. A. Vaughan, Electron Spin Relaxation Phenomena in Solids, Adam Hilger, London (1969)

[12] S. J. Bingham, Magnetic circular dichroism and electron paramagnetic resonance of transition ions, Ph.D. thesis, University of East Anglia (1993)

[13] C. P. Slichter, Principles of Magnetic Resonance, 1st ed., Harper, New York (1963)

[14] S. J. Bingham, J. Gutschank, B. Borger, D. Suter, A. J. Thomson, J. Chem. Phys., 113, 4331 (2000)

[15] R. Romestain, S. Geschwind, G. E. Devlin, P. A. Wolff, Phys. Rev. Letters, 33, 10 (1974)

[16] A. S. Borovik-Romanov, N. M. Kreines, Phys. Rep., 81, 351 (1982)

[17] R. Bernheim, Optical pumping, Benjamin, New York (1965)

[18] S. J. Bingham, B. Börger, D. Suter, A. J. Thomson, Rev. Sci. Instrum., 69, 3403 (1998)

[19] S. J. Bingham, D. Suter, A. Schweiger, A. J. Thomson, Chem. Phys. Letters, 266, 543 (1997)

[20] B. Börger, S. J. Bingham, J. Gutschank, M.-O. Schweika, D. Suter, A. J. Thomson, J. Chem. Phys., 111, 8565 (1999)

[21] S. J. Bingham, B. Börger, J. Gutschank, D. Suter, A. J. Thomson, JBIC, 5, 30 (2000)

[22] S. J. Bingham, J. J. Davies, D. Wolverson, Phys. Rev. B, 65, art. no. 155301 (2002) 
[23] L. C. Smith, D. Wolverson, S. J. Bingham, J. J. Davies, Physica Status Solidi BBasic Solid State Physics, 243, 892 (2006)

[24] L. C. Smith, S. J. Bingham, J. J. Davies, D. Wolverson, Appl. Phys. Lett., 87, Art. No. 202101 (2005)

[25] Y. R. Shen, The Principles of Nonlinear Optics, 1st ed., Wiley, New York (1984)

[26] H. G. Dehmelt, Phys. Rev., 105, 1924 (1957)

[27] A. Abragam, B. Bleaney, Electron Paramagnetic Resonance of Transition Ions, Oxford University Press, Oxford (1970)

[28] P. J. Stephens, Adv. Chem. Phys., 35, 197 (1976)

[29] T. H. Wilmshurst, Electron Spin Resonance Spectrometers, Adam Hilger, London (1967)

[30] K. W. Hipps, G. A. Crosby, J. Phys. Chem, 83, 555 (1979)

[31] W. R. Hagen, J. Mag. Res., 44, 447 (1981)

[32] F. Neese, W. G. Zumft, W. E. Antholine, P. M. H. Kroneck, J. Am. Chem. Soc., 118, 8692 (1996) 


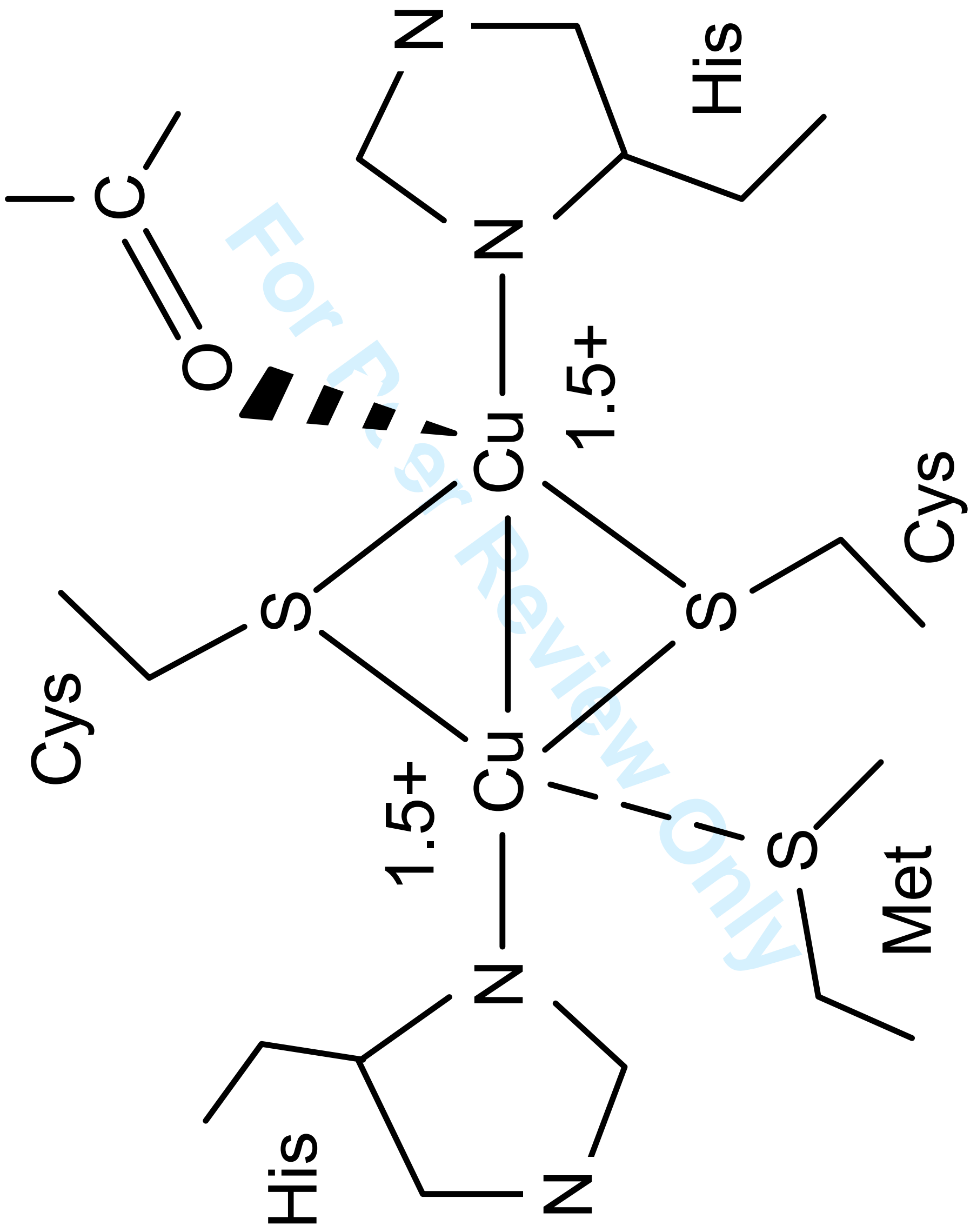




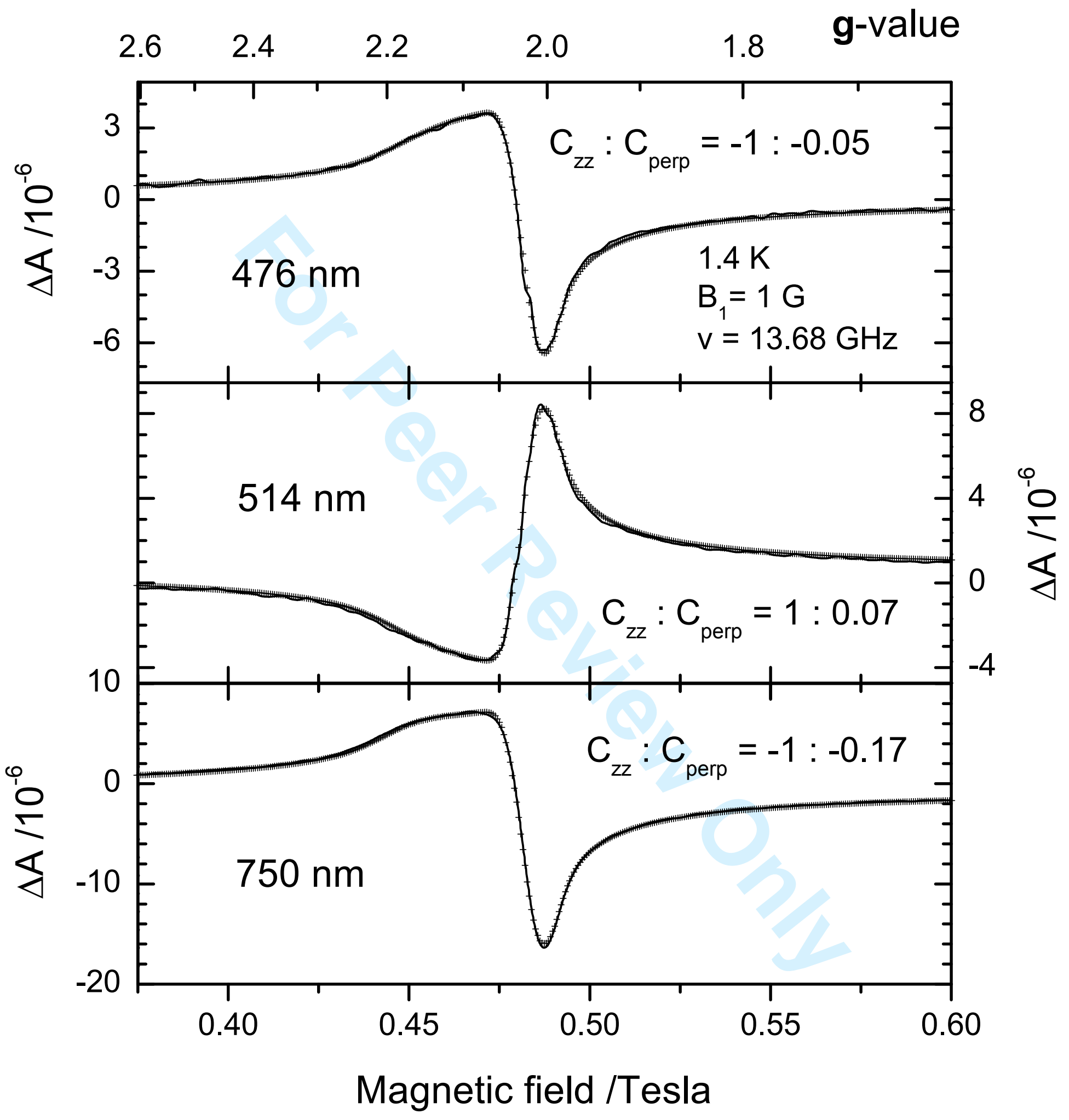

URL: http://mc.manuscriptcentral.com/tandf/tmph 


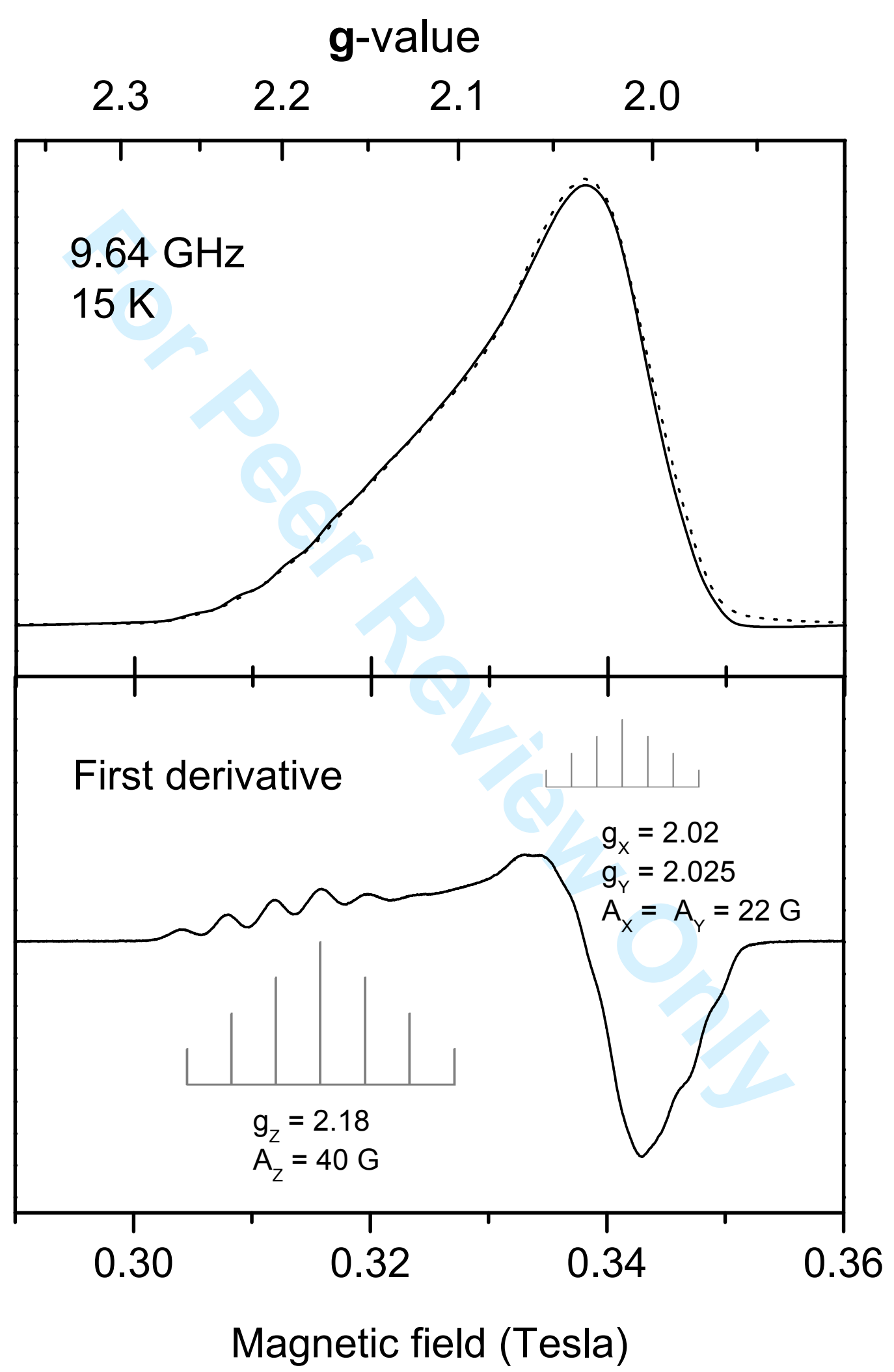

URL: http://mc.manuscriptcentral.com/tandf/tmph 


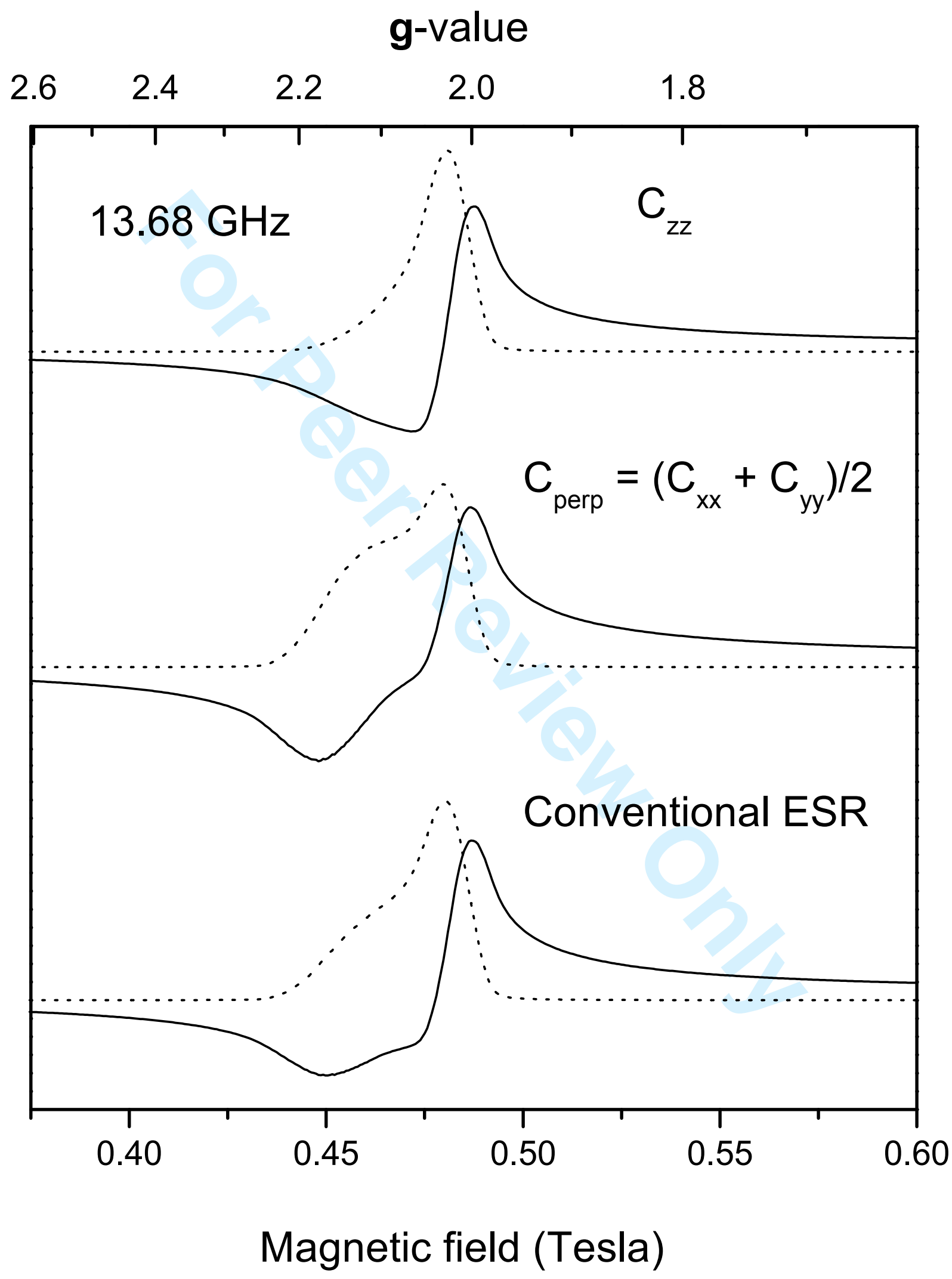

URL: http://mc.manuscriptcentral.com/tandf/tmph 